\title{
Socio-Cultural Knowledge in Conversational Inference
}

\author{
Xiaomei Yang \\ English Department, Qingdao University of Science \& Technology \\ Middle Section of Songling Road, Qingdao 266061, China \\ Tel: 86-532-8895-6956 E-mail: lindateacher@tom.com
}

\begin{abstract}
In addition to words and grammar, socio-cultural knowledge is also of vital importance in the interpretation of conversations. Socio-cultural knowledge helps participants to get contextualization cues and together with other signaling channels, participants will be able to perceive the context-bound information and prepare their appropriate responses. Socio-cultural knowledge is indispensable in our modern society where people have widely varying communicative and cultural backgrounds, which needs to be further studied.
\end{abstract}

Keywords: Socio-cultural knowledge, Contextualization cues, Conversational inference

\section{Introduction}

Recent studies of conversation from a variety of linguistic, psychological, anthropological and sociological perspectives have shed light upon a number of issues important to the study of conversational inference. It is generally agreed that grammatical and lexical knowledge are only two of several factors in the interpretation process. Aside from physical setting, participants' personal background knowledge and their attitudes towards each other, social-cultural assumptions concerning role and status relationships as well as social values associated with various message components also play an important role. In the following part, we will deal with the question of how social knowledge is stored in the mind, how it is retrieved from memory and how it interacts with grammatical and lexical knowledge in the act of conversing.

\section{Conversational Inference}

\subsection{Definition of Conversational Inference}

Conversational inference is the situated or context-bound process of interpretation, by means of which participants in an exchange assess other's intentions, and on which they base their response (Gumperz, 1982). The analysis of conversational inference follows such ongoing processes requires different and perhaps more indirect methods of study which examine not the lexical meanings of words or the semantic structure of sentences but interpretation as a function of the dynamic pattern of moves and countermoves as they follow one another in ongoing conversation.

Conversational inference is part of the very act of conversing. One indirectly or implicitly indicates how an utterance is to be interpreted and illustrates how one has interpreted another's utterance through verbal and nonverbal responses, and it is the nature of these responses rather than the independently determined meaning or truth value of individual utterances alone that governs evaluation of intent.

\subsection{Main Research of Interpretation of Conversational Inference}

Existing theories visualize the relationship of extra-linguistic, socio-cultural knowledge to grammar in one of two ways. The first is the anthropological tradition of ethnography of communication, where socio-cultural knowledge is seen as revealed in the performance of speech events defined as sequences of acts bounded in real time and space, and characterized by culturally specific values and norms that constrain both the form and the content of what is said. The second tradition of discourse analysis, deriving from speech act theory, linguistic pragmatics, frame semantics (Fillmore, 1977) and artificial intelligence posits abstract semantic constructs, variously called scripts, schemata, or frames, by means of which participants apply their knowledge of the world to the interpretation of what goes on in an encounter. The two traditions differ both in theory and in methodological approach.

\subsubsection{Conversation Analysis}

Sacks and his collaborators were the first systematically to focus on conversation as the simplest instance of a naturally organized activity, and attempt to study the process of conversational management only without making any 
assumptions about social and cultural background of participants. Their research concentrated on isolating strategies of effecting speaker change, opening and closing conversation, establishing semantic relations between utterances and so on.

One of Sack's key contributions to conversational analysis is his recognition that principles of conversational analysis inference are quite different from rules of grammar. The use of "maxim" suggests that interpretations take the form of preferences rather than obligatory rules. The point is that at the level of conversation, there are always many possible alternative interpretations, many more than exist at the level of sentence grammar. Choice among these is constrained by what the speaker intends to achieve in a particular interaction, as well as by expectations about the other's reactions and assumptions.

Conversation analysts were the first to provide systematic evidence for the cooperative nature of conversational processes and to give interactional substance to the claim that words have both relational and ideational significance. The perspective they have developed is therefore crucial to the study of verbal encounters. Yet still there are limitations in the way of analysis.

\subsubsection{The Limitation of Conversational Analysis}

The work of conversational analysis dose not account for the linguistic bases of conversational cooperation and in much of the empirical work of conversational analysis referential meanings that assume sharing of contextualization strategies are taken for granted. This view of language has serious limitations which affect both the validity of the analysts' attempts to capture participants' interpretive processes and the social import of their work. In order to account for inter-speaker differences in background knowledge, a socio-linguist needs to know how speakers use verbal skills to create contextual conditional that reflect particular culturally realistic scenes.

\section{Socio-culture Knowledge in Getting Conversational Inference}

\subsection{The Importance of Socio-culture Knowledge}

In order to account for inter-speaker differences in background knowledge, a socio-linguist needs to know how speakers use verbal skills to create contextual conditions that reflect particular culturally realistic scenes. How are speakers' grammatical and phonological abilities employed in this? Participants in a conversation must be able to scan phrases to predict when an utterance is about to end. They must be able to distinguish between rhetorical pauses and turn relinquishing pauses. Although overlap is an integral part of interaction, conversational cooperation required that interactional synchrony be maintained so that speakers cannot be interrupted at random. To follow the thematic progression of an argument, moreover, and to make one's contribution relevant, one must be able to recognize culturally possible lines of reasoning. It is therefore necessary to show how strategies of conversational management are integrated into other aspects of speakers' linguistic knowledge.

\subsection{Socio-culture Knowledge in Understanding Conversation}

\subsection{1 socio-culture knowledge and contextulization cue}

The linguistic basis for specific conversational exchange resides in con-occurrence expectations, which are learned in the course of previous interactive experience and form part of our habitual and instinctive linguistic knowledge. When reading novels, watching movies, or in real life, non-native speaker of English often have the feeling that they cannot get the exact meaning of the conversation even there is no problem of new words and grammar. Here is an example:

Example (1)

When I was sitting in an aisle seat on an airplane bound for Miami, Florida, I noticed two middle aged women walking towards the rear of the plane. Suddenly I heard from behind, "Tickets, please! Tickets, please!" at first I was startled and began to wonder why someone would be asking fro tickets so long after the state of the flight. Then one of the women smiled toward the other and said, "I told you to leave him at home." I looked up and saw a man passing the two women, saying, "step to the rear of the bus, please."

Native speakers will have no difficulty identifying this interchange as a joke, and hypothesizing that the three individuals concerned were probably traveling together and were perhaps tourists setting off on a pleasure trip. Here we will investigate what linguistic and other knowledge forms the basis for such inferences, and to what extent this knowledge is culturally specific.

The initial utterance, "Ticket, please," was repeated without pause and was spoken in higher than normal pitch, with more than usually loudness. For this reason it sounded like an announcement, or like a formulaic phrase associated with travel situations. The first inking that the interchange is a joke came with the woman's statement to her friend, "I told you to leave him at home." Although there is no way of knowing if the participants were looking at each other, the fact that the woman's statement was perfectly timed to follow the man's utterance was a cue that she was responding to him. Furthermore, the stress on "told" functioned to make her statement sound like a formulaic utterance, contributing to the 
hypothesis that she and he were engaging in a similar activity. If either the man or the woman had uttered their statements in normal pitch and conversational intonation, the connection between them might not have been clear. Only after people were able to hypothesize that the participants were joking, could they interpret their utterances. This hypothesis was then confirmed by the man's next statement, "Step into the rear of the bus, please." This was also uttered in announcement style. In retrospect, we may note that both of the man's utterances were formulaic in nature, and thus culturally specific and context bound. He was exploiting the association between walking down an aisle in a plane and the similar walk performed by a conductor on a train or a bus. In identifying the interaction as a joke, we were drawing on the same situational knowledge, as well as the fact that tourists bound for Miami are likely to engage in such joking.

Suprasegmental and other surface features of speech are often crucial to identifying what an interaction is about. When seen in isolation, sentences can have many intonation and paralinguistic contours, without change in referential meaning. The prevalent view is that these suprasegmental features add expressive overtones to basic meanings conveyed by core linguistic processes, that is, the signs by which listeners recognize these overtones tend to be seen as language-independent. The incident provides evidence for the claim that prosody is essential to conversational inference. The identification of specific conversational exchanges as representative of socio-culturally familiar activities is the process called "contextualization", by which we evaluate message meaning and sequencing patters in relation to aspects of the surface structure of the message, called "contextualization cue" (Gumperz, 1982). We regularly rely upon these matching processes in everyday conversation.

\subsubsection{Socio-cultural Knowledge and Other Signaling Channels}

Signaling of frames by a single speaker is not enough. All participants must be able to fit individual contributions into some overall theme roughly corresponding to a culturally identifiable activity, or a combination of these, and agree on relevant behavioral norms. They must recognize and explicitly or implicitly conform to others' expectations and show that they can participate in shifts in focus by building on others' signals in making their own contribution.

One common way in which conversational cooperation is communicated and monitored by participants is through what Yngve (1970) calls "back channel signals": interjections such as "OK", "right", "aha" or nods or other body movements. Other signs of cooperation are implied indirectly in the way speaker formulate response, i.e. in whether they follow shifts in style, agree in distinguishing from old or primary from secondary information, or in judging the quality of interpersonal relationships implied in a message, and know how to fill in what is implied but left unsaid or what to emphasize or de-emphasize, here is another example of how contextualization works and enters onto interpretation of intent.

\section{Example (2)}

The incident was observed at a luncheon counter, where the waitress behind the counter was talking with a friend seated at the counter.

Friend: I called Joe last night.

Waitress: You did? Well what'd he say?

Friend: Well, hi!

Waitress: Oh yeah? What else did he say?

Friend: Well he asked me out of course.

Waitress: Far out!

To participate in this exchange, the waitress, apart from having to rely on socio-culture schemata about dating situations, must recognize that the first statement, which seems complete on the surface, is actually the lead-in for a story that she is expected to help elicit. Further, she must know that "called" refers to a telephone call. She must know who Joe is; and she must realize that the call was no routine but had special meaning for her friend. Her reply "You did?" with exaggerated intonation contour and vowel elongation on "did", implicitly acknowledges all this. She then demonstrates that she has an idea of what is coming next in the story by her prompt "well what'd he say?"

The friend's response gives the main point of her story, but the meaning is not entirely conveyed by the content of what is said but by how it is said, which is communicated largely through prosody. In other words, participants must infer that the fall rise intonation on greetings such as "Hi" may signal surprise mixed with pleasure. Such intonation contours become meaningful through recurrent association with certain speech activities. Only if we know this, and are acquainted with the relevant conventions, can we interpret the speaker's use of "of course" in her subsequent comment.

\subsubsection{Socio-cultural Knowledge and Miscommunication}

From the above examples we can see that the signaling of speech activities is not a matter of unilateral action but rather of speaker-listener coordination involving rhythmic interchange of both verbal and nonverbal signs. If the participants 
in a conversation activity share different background expectations, there might be some inferential problems in the interpretation of a single message.

Example (3)

The incident took place in London, England, on a bus driven by a West Indian driver/conductor. The bus was standing at a stop, and passengers were filing in. The driver announced, "Exact change, please," as London bus drivers often do. When passengers who had been standing close by either did not have money ready or tried to give him a large bill, the driver repeated, "exact change, please." The second time around, he said "please" with extra loudness, high pitch, and falling intonation, and he seemed to pause before "please." One passenger so addressed, as well as others following him, walked down the bus aisle exchanging angry looks and obviously annoyed, muttering, "why do these people have to be so rude and threatening about it?"

In this case, requesting exact change is customary so that the accent on "change" would be expected. But here the politeness tag "please" is also accented and carries a falling tone. This goes counter to English prosodic conventions which associate falling tones with definiteness and finality, while rising tones, among other things, count as tentative and therefore tend to sound more polite. The interpretive effect here is that the setting off "please" spoken with a falling tone by contrast implies annoyance at something the listener did or is likely to do. The interpretation of rudeness is natural for listeners who rely on English contextualization convention to infer motivation.

Yet, in order to determine whether the conclusion that the driver was being rude corresponds to West Indian contextualization conventions, we need to look at how prosodic and paralinguistic cues normally function in West Indian conversation. According the researches of contextualization practice employed by London West Indians, their use of prosody and paralinguistics is significantly different from that of British English or American English speakers. The bus driver's accent on "please" can therefore be seen as an automatic consequence of tone grouping, not a matter of conscious choice. In addition, pitch and loudness differences do not necessarily carry expressive connotations. They are regularly used to indicate emphasis without any overtones of excitement or other emotions. From this example we can see miscommunication is very likely to be caused by different socio-cultural knowledge.

\section{The need of Deeper Study of Conversation}

How can we develop a more general theory of what accounts for both shared and culturally specific aspects of interpretive processes? Although ethnography of communication, discourse analysis and conversation analysis have made important contributions, we need some further study on the subtle elements that facilitate the progress of conversation. All verbal behaviors are governed by social norms specifying participant roles, rights and duties of each other, permissible topics, appropriate ways of speaking and way of introducing information. Such norms are context and network specific, so the notion of individuals relying on their own personal knowledge of the world to make sense of talk is an oversimplification. Sometimes the cognitive concept like the discourse analyst's schema is called for, but schemata cannot simply refer to knowledge of the physical world. In fact, some linguists (Gumperz, 1982) argued that a cognitive approach to discourse must build on interaction. It must account for the fact hat what is relevant background knowledge changes as the interaction progresses, that interpretations are multiply embedded and that, as Goffman (1974) has shown, several quite different interactions are often carried on at the same time. We can use "speech activity" (Levinson, 1979) for our present study goal.

A speech activity is a set of social relationships enacted about a set of schemata in relation to some communicative goal. Speech activities can be characterized through descriptive phrases such as "discussing politics", "chatting about weather", and "lecturing on linguistics". Such descriptions imply certain expectations about thematic progress, turn taking rules, form, and outcome of the interaction, as well as constraints on content. In the activity of discussing, we look for semantic relationships between subsequent utterances, and topic change is constrained. In the activity of chatting, topics change freely and no such expectations hold. Lecturing, in turn, implies clear role separating between speaker and audience and strong limitations on who can talk and what questions can be asked.

Although speech activities cannot be precisely listed, they are the means through which social knowledge is stored in the form of constrains on action and on possible interpretation. In verbal interaction social knowledge is retrieved through co-occurrence expectations of the type we have discussed. Distinctions among such activities as chatting, discussing and lecturing exist in all cultures, but each culture has its own constraints not only on content but also on the ways in which particular activities are carried out and signaled. Even within a culture, what one person would identify as "lecturing", another night interpret as "chatting with one's child", and so on.

Since speech activities are realized in action and since their identification is a function of ethnic and communicative background special problems arise in a modern society where people have widely varying communicative and cultural backgrounds, how can we be certain that our interpretation of what activity is being signaled is the same as the activity that the interlocutor has in mind, if our communicative background is not identical? So these are the problem needs further study in the future. 


\section{Conclusion}

To summarize, the conversational inference processes we have discussed involve several elements. On the one hand is the perception of contextualiztion cues. On the other is the problem of relating them to other signaling channels. Interpretation, in turn, requires first of all judges of expectedness and then a search for an interpretation. The linguistic character of contextualization cue is such that they are uninterpretable apart from concrete situations. To decide on an interpretation, participants must first make a preliminary interpretation, that is, they listen to a speech, form a hypothesis about what routine is being enacted, and then rely on social background knowledge and on co-occurrence expectations to evaluate what is intended and what attitudes are conveyed.

\section{References}

Fillmore, C. J. (1977). 'The case for case reopened', in P. Cole and J. Sadock (ed.), Syntax and Semantics. New York: Academic Press, Vol. 8, 59-81.

Goffman, E. (1974). Frame Analysis: an Essay on the Organization of Experience. Cambridge: Harvard University Press.

Gumperz, J. J. (1982). Discourse Strategies. Cambridge: Cambridge University Press.

Levinson, S. (1979). Activity Type and Language. Linguistics, 17, 365-99.

Sacks et al. (1974). A Simplest Systematics for the Organization of Turn-taking for Conversation. Language, 50, 696-735.

Yngve. (1970). Victor. On Getting a Word in Edge-wise. Chicago Linguistics Society, 6, 567-578. 